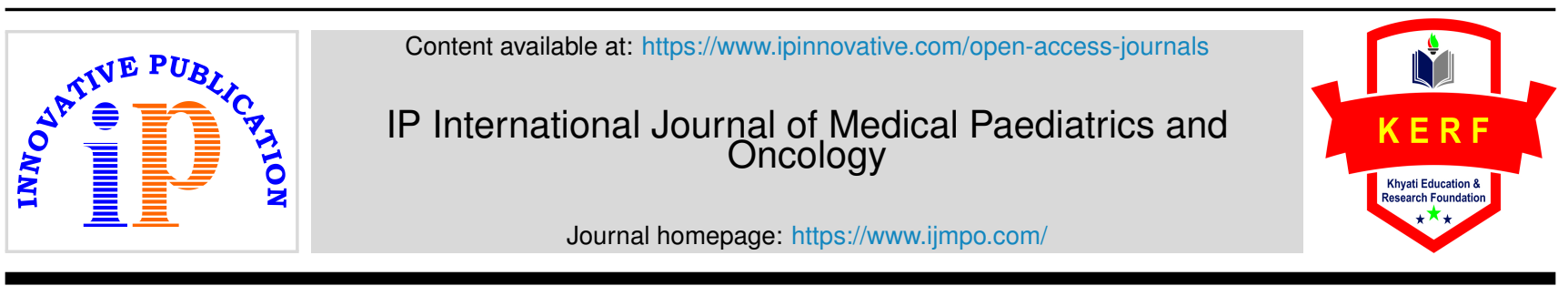

Original Research Article

\title{
To study the immunization status of children aged 1-5 years admitted to department of pediatrics, Lala Lajpat Rai \& Associated Hospitals, GSVM Medical College, Kanpur
}

\author{
Neeta Singh ${ }^{1}$,* \\ ${ }^{1}$ Dept. of Paediatrics, District Hospital, Shivpuri, Madhya Pradesh, India
}

\section{A R T I C L E I N F O}

\section{Article history:}

Received 16-06-2021

Accepted 23-06-2021

Available online 03-08-2021

Keywords:

Immunization

Children

Vaccines

\begin{abstract}
A B S T R A C T
Background \& Methods : The Present study was conducted in the Department of Pediatrics of Lala Lajpat Rai \& Associated Hospital GSVM Medical College, Kanpur. All the children aged between 1-5 years, admitted in Department of Pediatrics were asked to participate in this study. Information regarding vaccination, socio-demographic factors was collected from their parents and care takers. Accuracy and validity of information were confirmed by immunization card in possible situation and inspection for BCG scar.

Study Designed: Hospital based descriptive cross-sectional study.

Results: In upper class, $100 \%$ of children were completely immunized. In upper middle class, $80.8 \%$ children had complete and $19.1 \%$ partial immunization status. No one remained unimmunized in upper middle class. In lower middle class, $33.9 \%$ of children were completely immunized, $59.6 \%$ partially immunized and $6.4 \%$ remained unimmunized. Children who were 1 stin birth order, had maximum immunization coverage (44.8\%). Minimum immunization coverage was in birth order $>4(3.2 \%)$. P value is $<0.001$, indicates the significant relation between birth order and immunization status. As birth order increased, immunization coverage decreased.

Conclusion: In this present hospital based descriptive cross -sectional study, we found that $51.2 \%$ children were fully immunized, $45.6 \%$ were partially immunized and $3.2 \%$ children were unimmunized as per National immunization schedule. Children belonging to upper class were $100 \%$ completely immunized. In upper middle class $80.8 \%$ children in lower middle class $33.9 \%$, in upper lower class $55.7 \%$ and in lower class $50.9 \%$ children were completely immunized respectively. In lower class immunization coverage was higher than lower middle class. Droprate for BCG to pentavalent 1 was $10.64 \%$, similarly dropout from BCG to measles was $12.7 \%$. Dropout rate of pentavalent 1 to pentavalent 2 was $1.4 \%$, pentavalent 2 to pentavalent 3 was $2.6 \%$. It indicates that system is not able to hold the child once registered. Steps for improvement should focus on reducing the drop rate from BCG to pentavalent and measles.
\end{abstract}

(C) This is an open access article distributed under the terms of the Creative Commons Attribution License (https://creativecommons.org/licenses/by/4.0/) which permits unrestricted use, distribution, and reproduction in any medium, provided the original author and source are credited.

\section{Introduction}

Immunization is the process of inducing immunity against a specific disease. It can be done by the vaccines, immunoglobulins, and antisera. Immunity is of two types, active immunity and passive immunity. Active immunity is induced by administering a vaccine or toxoid. It stimulates

\footnotetext{
* Corresponding author.

E-mail address: medicalpub.2021@gmail.com (N. Singh).
}

the immune system to produce a prolonged humoral or cellular immune response. ${ }^{1}$

Immunization plays an important role in reducing morbidity and mortality. Last week of April (24th to 30th) is celebrated as world immunization week. ${ }^{2}$ Vaccination is one of the most powerful and cost-effective weapon of modern medicine to control infectious disease. Some infectious diseases whose control is solely based on immunization are polio, diphtheria, tetanus, measles etc. 
Unfortunately, we do not have a vaccine for every infectious disease like malaria and diarrheal diseases. ${ }^{3}$

Vaccines are defined as a whole or parts of microorganism administered to prevent an infectious disease. History of vaccine did not begin with Edward Jenner inventing a vaccine for smallpox. Evidence exists that Chinese used smallpox inoculation as early as $1000 \mathrm{BC}^{4}$. After smallpox, many other vaccines like Rabies, plague, cholera, typhoid, tuberculosis came in existence. A good vaccine is easy to administer, induces permanent immunity, free from toxic substances with minimal side effect and stable for a prolongeduse. ${ }^{5}$

Immunization is primary focus of child health and many programs have been launched by government of India for better coverage as immunization is a primary level prevention. $^{6}$

When immunization coverage reaches the figure of $80 \%$ or more, disease transmission pattern is severely disrupted and provides degree of protection even for remaining children who have not been immunized. This protection is because of "Herd Immunity". 7 Every country has its own immunization schedule. A well thought immunization schedule must be epidemiologically relevant, immunologically effective, operationally feasible and socially acceptable. Choice of vaccines in National immunization schedule is based on consideration of disease burden, vaccine availability and cost effectiveness. ${ }^{8}$ In May 1974 WHO launched first global immunization initiative EPI (expanded programme of immunization) aim to protect all children of world against 6 vaccine preventable diseases (VPDs)and was adopted by India in 1978.EPI chiefly covered urban areas. ${ }^{9}$

\section{Materials and Methods}

The Present study was conducted in the Department of Pediatrics of Lala Lajpat Rai \& Associated Hospital GSVM Medical College, Kanpur from Feb. 2017 to Oct. 2018. A total of 500 children were studied.

All the children aged between 1-5 years, admitted in Department of Pediatrics were asked to participate in this study. Information regarding vaccination, sociodemographic factors was collected from their parents and care takers. Accuracy and validity of information were confirmed by immunization card in possible situation and inspection for BCG scar.

\subsection{Data collection}

1. Informed oral written consent was taken from parents of the child.

2. All children aged 1-5 years admitted in the ward of our Hospital were studied using National immunization schedule and their parents were interviewed regarding awareness of immunization. The information was collected on pre-designed proforma and all the information was tabulated and interpreted through standard statistical methods and causes for partial and un-immunization were studied.

\subsection{Inclusion criteria}

All children aged 1-5 years admitted to Pediatrics ward

\subsection{Exclusion criteria}

1. Children $<1$ year and $>5$ years.

2. Children following IAP Schedule of immunization.

3. Children whose parents did not give consent for the study.

4. Children on long term steroid therapy.

\section{Results}

In our study, 500 children were included for assessment of immunization status. Subjects were distributed according to age group. Maximum subjects belonged to 1-2 years 274 $(54.8 \%)$ and maximum immunization was found among children between 1 to 2 years of age (58.7\%). Table 1

Value $<0001$ indicates a significant association between age and immunization status

Among 500 subjects studied, $256 \quad$ (51.2\%) were completely immunized, $228(45.6 \%)$ were partially immunized and $16(3.2 \%)$ were unimmunized.Table 2

Among 500 study subjects, 423 (84.6\%) had vaccination card and $77(15.4 \%)$ had no vaccination card.Table 3

In upper class, $100 \%$ of children were completely immunized. Table 4

In upper middle class, $80.8 \%$ children had complete and $19.1 \%$ partial immunization status.

No one remained unimmunized in upper middle class.

In lower middle class, $33.9 \%$ of children were completely immunized, $59.6 \%$ partially immunized and $6.4 \%$ remained unimmunized.

In upper lower class, $55.7 \%$ children were completely immunized $42 \%$ partially immunized, and $2.2 \%$ were unimmunized.

In lower class, $50.9 \%$ were completely immunized, $49 \%$ partially immunized and no one was unimmunized.

Value $<0$ 001, indicates significant relation between socio-economic status and immunization status

Children who were $1^{s t}$ in birth order, had maximum immunization coverage $(44.8 \%)$. Minimum immunization coverage was in birth order $>4(3.2 \%)$. $\mathrm{P}$ value is $<0.001$, indicates the significant relation between birth order and immunization status. As birth order increased, immunization coverage decreased.Table 5 
Table 1: Age distribution of the study subjects

\begin{tabular}{lcccc}
\hline Age group & $\mathbf{N}=\mathbf{5 0 0}$ & Percentage & Complete & \% \\
1-2 year & 274 & $54.8 \%$ & 161 & 58.7 \\
2-3 year & 78 & $15.6 \%$ & 33 & 42.3 \\
3-4 year & 49 & $9.8 \%$ & 15 & 30.6 \\
4-5 year & 99 & $19.8 \%$ & 47 & 47.4 \\
\hline
\end{tabular}

Table 2: Immunization status in study subjects

\begin{tabular}{lcc}
\hline & N=500 & Percent \\
Completely immunized & 256 & $51.2 \%$ \\
Partially immunized & 228 & $45.6 \%$ \\
Unimmunized & 16 & $3.2 \%$ \\
\hline
\end{tabular}

Table 3: Distribution of vaccination card among study subjects.

\begin{tabular}{lcc}
\hline Vaccination Card & N=500 & Percent \\
Available & 423 & 84.6 \\
Not available & 77 & 15.4 \\
\hline
\end{tabular}

Table 4: Distribution of immunization status according to socio-economic status.

\begin{tabular}{|c|c|c|c|c|c|c|}
\hline Class & Complete & $\%$ & Partial & $\%$ & Unimmunized & $\%$ \\
\hline Upper & 12 & 100 & 0 & 0 & 0 & 0 \\
\hline Upper Middle & 38 & 80.8 & 9 & 19.1 & 0 & 0 \\
\hline Lower Middle & 58 & 33.9 & 102 & 59.6 & 11 & 6.4 \\
\hline Upper Lower & 122 & 55.7 & 92 & 42 & 5 & 2.2 \\
\hline Lower & 26 & 50.9 & 25 & 49 & 0 & 0 \\
\hline
\end{tabular}

Table 5: Distribution of immunization status according to birth order.

\begin{tabular}{lcccccccc}
\hline Birth Order & Complete & \% & Partial & \% & Unimmunized & \% & Total & \% \\
$1^{\text {st }}$ & 123 & 54.9 & 96 & 42.8 & 5 & 5.2 & 224 & 44.8 \\
$2^{\text {nd }}$ & 91 & 54.8 & 70 & 42.1 & 5 & 3 & 166 & 33.2 \\
$3^{\text {rd }}$ & 31 & 43.6 & 40 & 56.3 & 0 & 0 & 71 & 14.2 \\
$4^{\text {th }}$ & 11 & 47.8 & 12 & 52.1 & 0 & 0 & 23 & 4.6 \\
$>4$ th & 0 & 0 & 10 & 62.5 & 6 & 37.5 & 16 \\
\hline
\end{tabular}

\section{Discussion}

The Present study was conducted in tertiary care center of Kanpur from Feb. 2017 to Oct. 2018 with study population of 500 children belonging to 1-5 years age group.

Among them, 256 (51.2\%) Children were fully immunized. Similar findings were noted in studies of Ashish Awasthi et al. (57.03\%). ${ }^{10}$

Suresh Kumar Verma et al. (48.5\%), ${ }^{11}$ Anil B. Kurane et al. $(65.2 \%){ }^{12}$ and While Mathew et al. (25\%). Nirupam et al $(34.5 \%)^{13}$ noted very low percentage of immunization.

The NFHS - 4 data showed that the percentage of fully immunized children in Kanpur Nagar was $50.9 \%$ and for UP $(51.1 \%)$. Our result for fully immunized children was similar to NFHS 4 survey.

$45.6 \%$ Children were partially immunized in the study population. Similar findings were seen in study conducted by Kumar et al. (48\%), ${ }^{14}$ Jain et al. (44.5\%), Anil B. Kurane et al. $(34 \%) .{ }^{12}$ In present study, $3.2 \%$ children were un- immunized which was similar to Prabhakaran Nair et al. ${ }^{15}$ $(4.2 \%)$

In this study, $303(60.6 \%)$ children were males and 197 $(39.4 \%)$ were females whereas in the study of Kumar et al. $(58.6 \%)^{14}$ children were male and $41.6 \%$ were female. Similar results were shown in study of Deshmukh PR et al. ${ }^{7}$ (52\% Male and $48 \%$ Female).

\section{Conclusion}

In this present hospital based descriptive cross -sectional study, we found that $51.2 \%$ children were fully immunized, $45.6 \%$ were partially immunized and $3.2 \%$ children were unimmunized as per National immunization schedule.

Children belonging to upper class were $100 \%$ completely immunized. In upper middle class $80.8 \%$ children in lower middle class $33.9 \%$, in upper lower class $55.7 \%$ and in lower class $50.9 \%$ children were completely immunized respectively. In lower class immunization coverage was 
higher than lower middle class.

Droprate for BCG to pentavalent 1 was $10.64 \%$, similarly dropout from BCG to measles was $12.7 \%$. Dropout rate of pentavalent 1 to pentavalent 2 was $1.4 \%$, pentavalent 2 to pentavalent 3 was $2.6 \%$. It indicates that system is not able to hold the child once registered. Steps for improvement should focus on reducing the drop rate from BCG to pentavalent and measles.

\section{Conflict of Interest}

The authors declare that there are no conflicts of interest in this paper.

\section{Source of Funding}

None.

\section{References}

1. Park K Principles of Epidemiology and epidemiological method. Text Book of Preventive and Social Medicine Jabalpur. In: Banarsidas Bhanot publishers 24th Edn. WHO; 1982. p. 461.

2. Kunal K, Vaisanav J. Evaluation of Immunization Status: A Hospital Based Study of 1000 Children at Department of Paediatrics BMC, Vadodara. Paripex- Indian J Res. 2016;5(8):382-5.

3. Sinha A, Singh S. 3. Ghai textbook of essential pediatrics gth edition CBS publishers and distributors Pvt. Ltd. Immunization and immunodeficiency.

4. Frenkel LD, Nielson K. Immunization issues for the 21 Century Ann Allergy. Asthma Immunol. 2003;90(3):45-52.

5. Jain SK, Chawla U, Gupta N, Gupta RS, Venkatesh S, Lal S, et al. Child survival and safe motherhood program in Rajasthan. Indian $J$ Pediatr. 2006;73(1):43-7. 100:-101007/6+02758259.

6. Prabhakaran TN, Varughese E. Immunization coverage of infantsRural-Urban difference in Kerala. Indian Pediatr. 1994;31:139-43.

7. Deshmukh PR, Dongre AR, Gupta SS, Garg BS. Newly developed WHO growth standards: Implications for demographic surveys and child health programs. Indian J Pediatr. 2007;74(11):987-90. doi:-10.007/s]2098-007-0) $81-0$

8. Rajeev V, Radhamani V. A cross sectional study on immunization status of anganwadi children in a rural area of north Kerala, India. Int J Res Med Sci. 2016;4(6):2039-43. 100:10.18203/2320日 6012.1]rms20161756

9. Kadri AM, Singh A, Jain S, Mahajan RG, Trivedi A. Study on immunization coverage in urban slums of Ahmedabad city. Health Popul: Perspect. 2010;33(1):50-4.

10. Awasthi A, Pandey CM, Singh U, Kumar S, Singh TB. Maternal determinants of immunization status of children aged 12-23 months in urban slums of Varanasi, India. Clin Epidemiol Global Health. 2015;3(3):110-6. 001:10.1016/].cegh.2014.07.004.

11. Verma SK, Yadav A, Dabi DR. A Hospital Based Study of Assessment of Missed Opportunities of Immunization in Children Aged Less Than Two Years. J Pediatr Neonatal Care. 2017;6(5):00261. 01:10.15406/jpnc.2017.06.00261.

12. Kurane AB, Swathi D. A study of immunization status of children in the age group 2-5 years. Int J Contemp Pediatr. 2018;5(3):922-7. do1:10.18203/2349-3291.1]cp20181514.

13. Nirupam S. UIP in Sarojini Nagar block of Lucknow district. Indian J Comm Med. 1991;16(2):82-4.

14. Sagar N, Kashyap V, Kumar M. Study on Immunization Status among Children's between 2 To 6 Years of Age Group in Rural Field Practices Area of Ormanjhi of Rims. Ranchi IOSR J Dent Med Sci. 2016;15(1):108-12. 10i: $109790 / 0853-15310812$.

15. Prabhakaran TN, Varughese E. Immunization coverage of infantsRural-Urban difference in Kerala. Indian Pediatr. 1994;31:139-43.

\section{Author biography}

Neeta Singh, PGMO Paediatrics

Cite this article: Singh N. To study the immunization status of children aged 1-5 years admitted to department of pediatrics, Lala Lajpat Rai \& Associated Hospitals, GSVM Medical College, Kanpur. IP Int J Med Paediatr Oncol 2021;7(2):63-66 